\title{
How much more can sunspots tell us about the solar dynamo?
}

\author{
Aimee A. Norton ${ }^{1}$, Eric H. Jones ${ }^{2}$, Y. Liu ${ }^{1}$, \\ K. Hayashi ${ }^{1}$, J. T. Hoeksema ${ }^{1}$ and Jesper Schou ${ }^{1}$ \\ ${ }^{1}$ HEPL, Solar Physics, Stanford University, \\ CA 94305 USA, \\ email: aanorton@stanford.edu \\ ${ }^{2}$ Centre for Astronomy, University of Southern Queensland, \\ Toowoomba, QLD, Australia \\ email: eric.jones@jcu.edu.au
}

\begin{abstract}
Sunspot observations inspired solar dynamo theory and continue to do so. Simply counting them established the sunspot cycle and its period. Latitudinal distributions introduced the tough constraint that the source of sunspots moves equator-ward as the cycle progresses. Observations of Hale's polarity law mandated hemispheric asymmetry. How much more can sunspots tell us about the solar dynamo? We draw attention to a few outstanding questions raised by inherent sunspot properties. Namely, how to explain sunspot rotation rates, the incoherence of follower spots, the longitudinal spacing of sunspot groups, and brightness trends within a given sunspot cycle. After reviewing the first several topics, we then present new results on the brightness of sunspots in Cycle 24 as observed with the Helioseismic Magnetic Imager (HMI). We compare these results to the sunspot brightness observed in Cycle 23 with the Michelson Doppler Imager (MDI). Next, we compare the minimum intensities of five sunspots simultaneously observed by the Hinode Solar Optical Telescope Spectropolarimeter (SOT-SP) and HMI to verify that the minimum brightness of sunspot umbrae correlates well to the maximum field strength. We then examine 90 and 52 sunspots in the north and south hemisphere, respectively, from 2010 - 2012. Finally, we conclude that the average maximum field strengths of umbra 40 Carrington Rotations into Cycle 24 are 2690 Gauss, virtually indistinguishable from the 2660 Gauss value observed at a similar time in Cycle 23 with MDI.
\end{abstract}

Keywords. Sunspots, dynamo, magnetic fields

\section{Introduction}

Sunspots are solar features whose ease of observation makes them historically one of the best-observed astrophysical phenomenon, being famously observed in the West by Galileo in 1612 and observed in other cultures, such as China, as early as 364 BC. Any acceptable solar dynamo model or theory must be able to explain the following characteristics of the solar cycle: the number of sunspots increasing then decreasing over a period of approximately 11 years, the majority of sunspots appearing in pair with opposite polarities, polarities of the leading spots with respect to rotation being antisymmetric across the equator, activity belts within \pm 35 degrees of the equator with sunspots appearing closer to the equator as time progresses.

One type of dynamo scenario proposed to operate in the Sun is a flux-transport Babcock-Leighton dynamo (Babcock, 1961; Leighton, 1964) in which toroidal fields are generated by shearing at depth in the tachocline, poloidal fields are generated at the surface by the decay of tilted sunspot groups. In the flux-transport Babcock-Leighton dynamo, the transport of flux towards the poles at high latitude, the transport of sunspot 


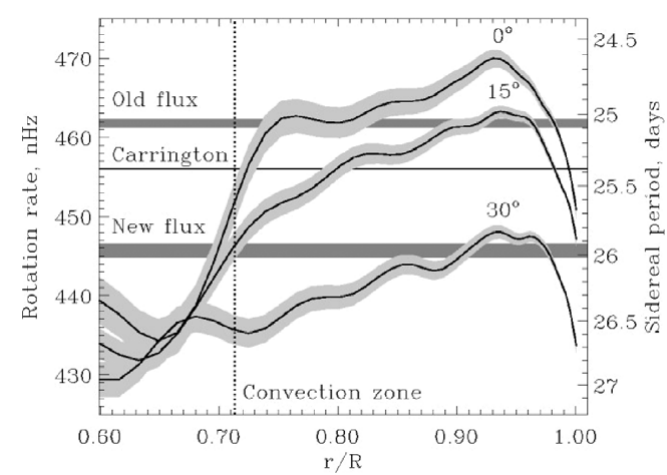

Figure 1. Reproduction from Benevolenskaya et al. (1999) in which surface rotation rates of old and new cycle flux observed with MDI (horizontal bars) are compared to the Carrington Rotation and helioseismological internal rotation rates at three latitudes (Schou et al. 1998). New cycle flux near $30^{\circ}$ rotates slower than old flux near $5^{\circ}$. Old cycle flux rotates faster than the average tachocline rotation rate.

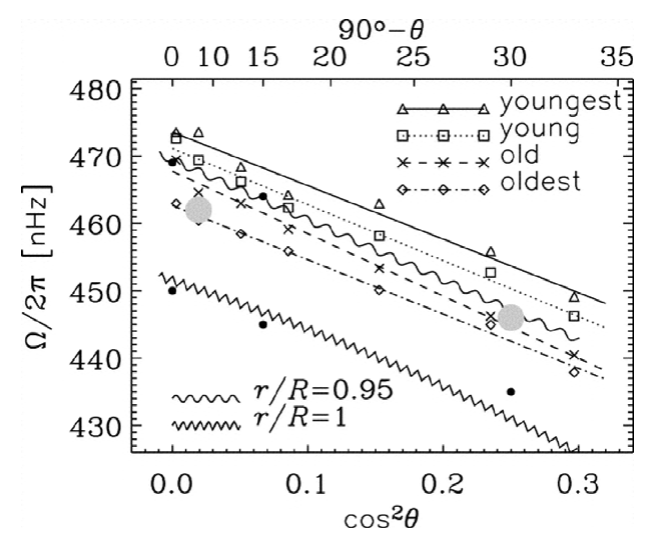

Figure 2. Reproduction of a figure from Brandenburg (2005) in which the rotation rate of sunspots of different ages as a function of colatitude $(\theta)$; compared with the internal angular velocity at $\mathrm{r}=0.95 \mathrm{R}_{\odot}$ determined by helioseismology and the surface rotation rate. The large gray circles at about $30^{\circ}$ and $4^{\circ}$ denote the angular velocities of active zones near the beginning and the end of the cycle, respectively.

flux towards the equator, and the cycle length is set by the rate of a meridional flow cell operating in each hemisphere moving poleward at the surface and equatorward at depth. In contrast, another popular type of dynamo model is the near-surface shear dynamo, also called the distributed dynamo since the toroidal magnetic field is thought to be generated throughout the convection zone. In this model, the near surface shear layer conditions allow equatorward flow of magnetic flux.

As observers, we are asking: what surface magnetic field signatures could distinguish between proposed dynamo mechanism? Open questions that could be addressed by surface magnetic field observations are:

- Does the rotation rate of sunspots indicate "anchor depth"?

- How can we interpret the incoherence of follower sunspots?

- Does the longitudinal distribution of sunspots indicate that giant cells play a role in their formation?

- What trends are seen in sunspots through the solar cycle that provide insight into their generation?

We summarize and review previous work on these topics while looking at the surface magnetism of Cycle 24 with HMI for indications as to whether the dynamo operating in the Sun has more in common with a deep-seated Babcock-Leighton dynamo or a near-surface shear layer dynamo.

\section{Rotation Rate of Sunspots}

The rotation rate of the plasma inside the Sun varies with latitude and depth. The tachocline is the name given to a thin shell inside the Sun, at the base of the convection zone, where the rotation pattern changes most distinctly. Deeper than the tachocline the solar rotation is uniform, whereas above the tachocline, the rotation rate varies with latitude. The rate is faster at the equator than at the poles. Helioseismology has provided 


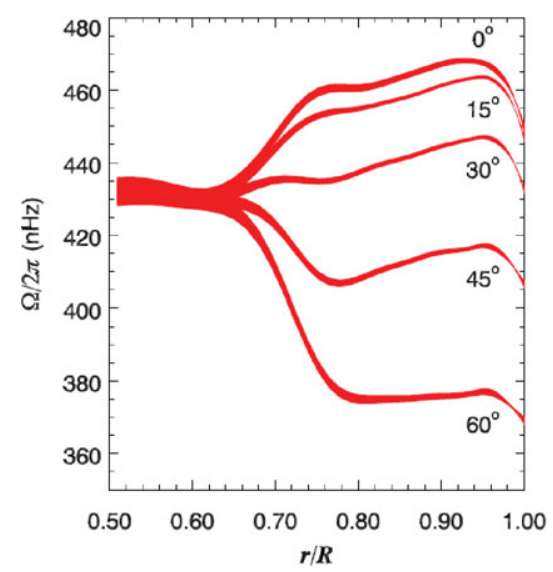

Figure 3. The internal solar rotation as a result of helioseismology. The rotation rate is shown for several latitudes as a function of depth using GONG data from 1995-2009. The tachocline is the layer around $\mathrm{r} / \mathrm{R} \approx$ 0.7 where the transition occurs to uniform rotation. Graph courtesy of NSF's National Solar Observatory.

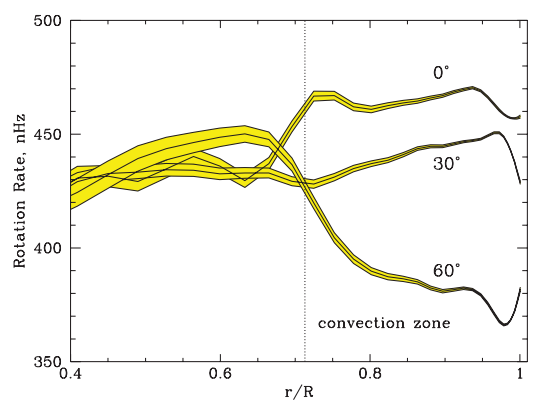

Figure 4. Figure courtesy of Kosovichev et al.(1997) showing the rotation rate as a function of depth in the solar interior. Three latitudes are shown as determined from MDI medium- $l$ data. The width of the line indicates uncertainty.

the angular velocity distribution inside the Sun as shown by the curved lines in Figure 1 (Kosovichev et al. 1997; Schou et al. 1998; Wilson et al. 1997).

Does the rotation rate of sunspots indicate anchor depth? Sunspots rotate faster than the surface plasma at the same latitude (Benevolenskaya et al., 1999). In Figure 1, all rates can be understood by anchoring at $0.97 \mathrm{R}_{\odot}$ lending support to the near-surface dynamo model. However, the interpretation is redundant for old cycle flux since a similar rate is observed near $0.71 \mathrm{R}_{\odot}$. The expected range of rotation rates near the base of the convection zone for sunspot latitudes is $435-455 \mathrm{nHz}$ while near the surface layer it is 445-470 nHz, meaning there is redundancy in interpretating a portion of the range of values. Figure 2 shows that smaller and younger sunspots rotate faster than larger, older spots at a comparable latitude (Brandenberg et al., 2005). In this case, young and old refer to how long an individual spot has been on the solar disk in terms of days, not old cycle flux as in Figure 1. Note that any indication of flux anchored at a certain depth need not imply it was generated there.

One question that remains, and is important in understanding the rotation rates of active regions, is: what does the near surface layer $\left(\begin{array}{llll}0.95 & 1.0 & \mathrm{R}_{\odot}\end{array}\right)$ rotation look like at high latitudes? Notice that Figure 3 (courtesy of National Solar Observatory) and Figure 4 (Kosovichev et al., 1997) show different trends for the rotation rate of the plasma at $60^{\circ}$ from $0.95-1.0 \mathrm{R}_{\odot}$. There is still some debate on this topic - if radial shear near the surface remains negative at high latitudes, and near surface dynamo assumes no meridional circulation necessary for sunspot migration, why do sunspots only appear near the equator? This is an open question that can be addressed by helioseismology and active region studies.

A serious problem in sunspot rotation studies is the method often used to remove differential rotation. A pair, or group, of sunspots are usually tracked at a single rotational velocity, corresponding to the latitude of the area weighted, center-of-gravity of the entire group. This can impart relative drifts and fake proper motions between the lower and higher latitude spots (Petrovay, 1993). Petrovay (1993) argues that the motion of large 
flux ropes is largely independent from the plasma, and therefore should not be used as a indication of anchor depth. However, one of the strongest arguments being put forth in favor of a near-surface dynamo mechanism distributed throughout the convection zone is the observed rotation rates of sunspots. We leave it to the readers to form their own opinions on the meaning of sunspot rotation rates and cautiously apply area-weighting for any studies.

\section{Follower Sunspot Incoherence}

Observations of sunspot groups show that the preceding sunspots within a group are generally larger and live longer than follower sunspots. Follower sunspots are less coherent; they are more dispersed and fragmented (Bray \& Loughhead, 1964; Fan, 2009). Fan et al., 1993 were able to reproduce this morphological asymmetry using thin flux tubes with relatively weak initial field strengths $\left(\sim 10^{4} \mathrm{G}\right)$. Subsequent work by Caligari et al., 1995 and Caligari et al., 1998, however, demonstrated that in order to better reproduce the observed properties of active regions, a greater initial field strength $\left(\sim 10^{5} \mathrm{G}\right)$ is required. At this greater field strength, the observed incoherence in the follower spots isn't reproduced. Stein \& Nordlund (2012) show that magneto-convection simulations with the STAGGER code (Beeck et al., 2012) can produce flux concentrations at the solar surface similar to active regions without the need to introduce a coherent flux tube at the bottom of their simulation domain. They use a computational domain $20 \mathrm{Mm}$ deep by 48 $\mathrm{Mm}$ wide and show follower spots to be less coherent than leading ones. However, these simulations do not yet reproduce penumbra and other observed active region features, indicating that the simulations are incomplete as yet. In summary, there are problems in both the deep-seated and near-surface dynamo models in explaining the incoherence and instability of follower sunspots. This topic deserves more attention.

\section{Does the longitudinal distribution of sunspots indicate that giant cells play a role in their formation?}

The sunspot size scale is commonly quoted as being $\approx 100 \mathrm{Mm}$, meaning that the distance covered by any given sunspot pair is about this size. We are interested in the average separation between sunspot groups. Since the detection of giant cells (Beck, Duvall, \& Scherrer, 1998) extending over $40-50^{\circ}$ degrees in longitude, it has seemed possible that flows associated with giant cells either pin down flux (discouraging flux from rising) at the downflow lanes or encourage a buoyant instability at the center of the giant cell where upflow occurs. This notion seems a bit fantastical until one looks at the spacing in between sunspot groups in the sunspot cycle. By eye, it does indeed appear that there is often a $40^{\circ}$ spacing between active region groups. If the giant cells were influencing sunspot locations, then we would expect a size scale of $\approx 480 \mathrm{Mm}$, or $m=9$, in active longitudes.

To quantify this notion, we analyzed HMI magnetic field synoptic map data for Carrington Rotations 2097-2126 (30 rotations in all), translating the maps into a simplistic format where all points on the map were represented by zeros if no sunspots were present. If sunspots were present, a single value of one represented the center of the umbra location. We then removed the latitude axis, in essence creating a 1-dimensional binary array for each Carrington rotation, so that only the sunspot spacing in longitude was recorded. Taking a spatial Fourier transform of each Carrington rotation and adding all power spectra showed significant power at $m=6$ and $m=43$, corresponding to scales of roughly 700 and $100 \mathrm{Mm}$. The $100 \mathrm{Mm}$ is the spacing between two sunspots within 
the same group, but the $700 \mathrm{Mm}$ signal was a surprise to us and is not understood. Due to page limits, we do not reproduce the power spectrum in this paper. Nevertheless, it would be interesting to do further analysis, especially to determine if sunspot locations correlated with the center or lanes of giant cell or Rossby wave patterns.

\section{Brightness Ratio Trends in Sunspots over the Solar Cycle}

The existence of definitive trends within a given sunspot cycle for average or maximum sunspot magnetic field strength, area, brightness and inferred temperature has been a point of contention for decades. Sunspot umbrae, in particular the darkest parts the dark nuclei that evolve the least during sunspot formation and dissipation (Socas-Navarro 2003), may provide a diagnostic of the dynamo mechanism. This should be true if the flux formation and eruption process determines the peak magnetic field strength of sunspots. One of the reasons the intensity of sunspots is interesting is that the Brightness Ratios (BR), the ratio of the continuum intensity in the darkest part of the sunspot sunspot umbra to the average surrounding continuum intensity of the photosphere, is a very good proxy for magnetic field strength.

$$
B R=\frac{\text { minimum }\left(I_{c} \text { umbra }\right)}{\text { average }\left(I_{c} \text { photosphere }\right)}
$$

The BR is free from many problems experienced in the inversion of Stokes profiles to determine field strength and therefore is useful in inferring field strengths over several solar cycles using different telescopes with varied spectral lines. Martínez Pillet \& Vázquez (1993) studied the distribution of continuum intensity and magnetic field in eight umbrae and demonstrated that the smaller continuum intensities corresponded to larger field strengths. This relationship can be understood in terms of the sunspot response to a horizontal force balance. Stronger umbral magnetic fields (higher magnetic pressure) need less plasma (lower gas pressure) to balance the external forces. The local relationship between temperature and magnetic field in sunspot umbrae dictates that the darker, cooler umbrae contain stronger magnetic fields. A brief literature review of intensity trends in sunspots is as follows.

Maltby et al. (1986) and Albregtsen \& Maltby (1978) studied the variations of umbral intensities in 22 sunspots and found that umbrae became progressively brighter during solar cycles 20 and 21, with a calculated temperature increase of $300 \mathrm{~K}$ from the beginning to the end of each cycle. Norton \& Gilman (2004) studied 650 sunspots observed with MDI to look for trends from 1998-2003 (Cycle 23). A decrease (not linear) in brightness was seen until sunspot maximum in the North, then sunspots began to brighten again with a maximum difference of $650 \mathrm{~K}$ was found in the $\mathrm{N}$ hemisphere. Mathew et al. (2007) studied 160 sunspots from 19982004 in Cycle 23 using MDI data. They reported no trend in umbral brightness. They limited their data to sunspots with radii between 5-15 arcsec and they corrected for stray light. Rezaei, Beck \& Schmidt (2012) studied 183 sunspots from 19992011 with Tenerife Infrared Polarimeter. They find a trend that sunspots in the late stage of cycle 23 are weaker than those at the start of the cycle. The decrease in the field strength with time is about 94 Gauss/yr while the continuum intensity increased linearly with a rate of $1.3 \%$ of Ic/yr from max to min, while the umbral area did not show any trend. Sunspots in the new Cycle 24 show higher field strengths and lower continuum intensities than those at the end of cycle No. 23, interrupting the trend. Penn \& Livingston (2006) observed over 900 sunspot umbrae from 1998-2005 (Cycle 23) using the Fe I $1564.8 \mathrm{~nm}$ line at NSO McMath Pierce. They find that the maximum sunspot magnetic fields decreased at about 52 Gauss/yr. and that the umbrae 

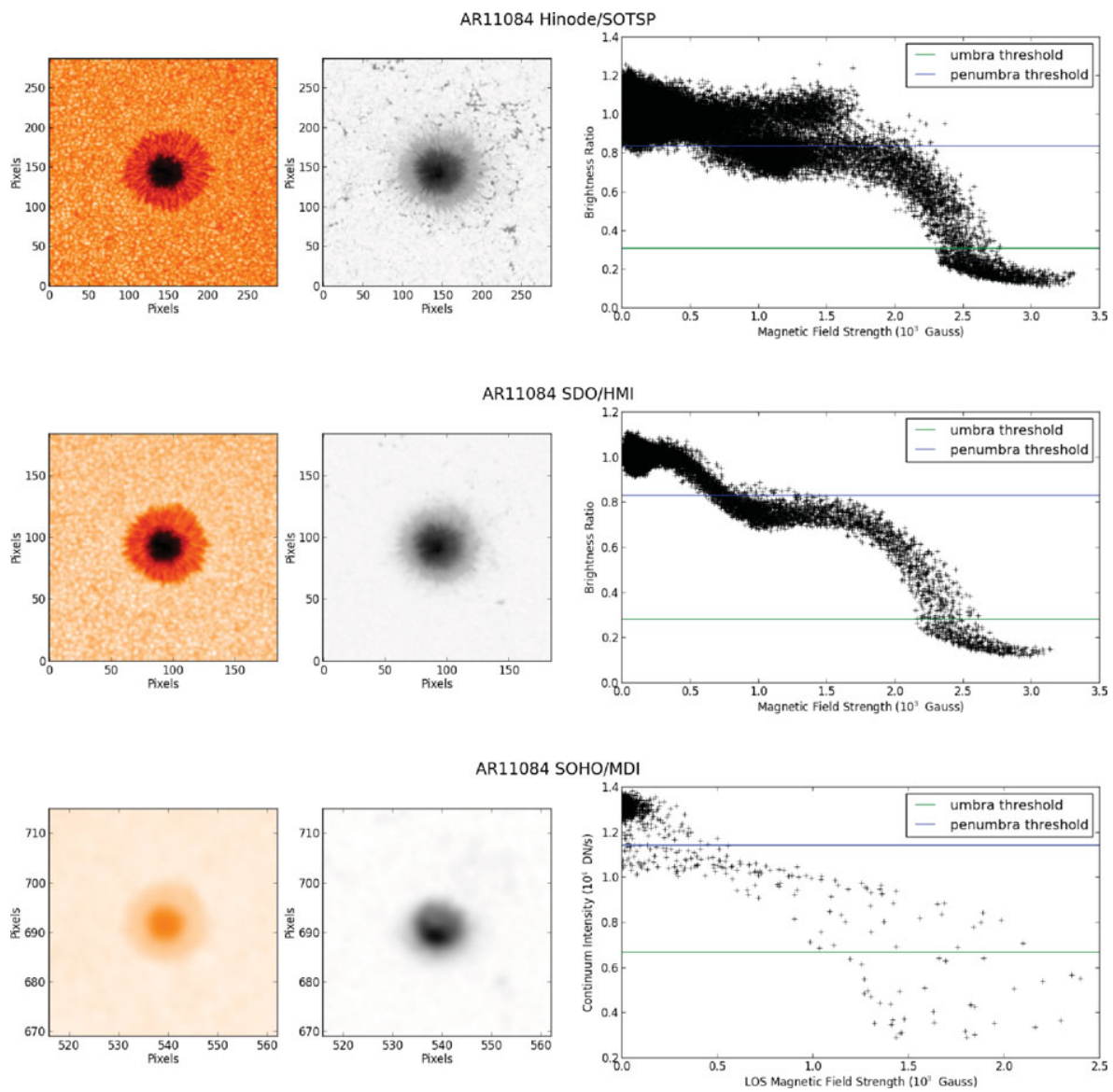

Figure 5. Continuum image (left), Magnetogram (center), and Brightness Ratio vs. Magnetic Field Strength (right) for Active Region 11084, as seen by Hinode SOT-SP (top), SDO HMI (middle) and SOHO/MDI (bottom).

became linearly brighter with the normalized umbral intensity changing from 0.60 to 0.75 (corresponding to a rise of 550 Kelvin). Watson, Fletcher \& Marshall (2011) analyze over 30000 sunspot observations using MDI and find a cyclical trend that follows sunspot number (not linear) with sunspots having stronger maximum magnetic fields at sunspot maximum and weaker fields at sunspot minimum. In summary, 5 out of 6 of these past studies agree that sunspots are darker at sunspot maximum than later in the same cycle. What is not agreed upon is whether the earliest part of the cycle produces spots lighter or darker than those at sunspot maximum. Note that the ascending phase of the cycle is the shortest and the least well-sampled.

To compare Cycle 23 observed by MDI to Cycle 24 observed by HMI, we must first understand how to compare the instrument data. HMI is a filter-based instrument observing Stokes I, Q, U and V at six wavelength positions across the $6173 \AA$ Fe-I line with 76 mA filter space sampling (Scherrer et al., 2012; Norton et al., 2006). The HMI pipeline uses a Milne-Eddington inversion code named the Very Fast Inversion of the Stokes Vector (VFISV) (Borrero et al., 2011; Borrero et al., 2007) to determine the solar atmospheric parameters based on Stokes observations. MDI is a filter-based instrument sampling the $6768 \AA$ Ni-I line at 5 wavelengths. MDI only sampled Stokes I and V using 
Table 1. Active regions with associated observation dates, HMI and SOT-SP brightness ratios, magnetic field strengths, and $\mu$ values

\begin{tabular}{|c|c|c|c|c|c|c|}
\hline Active Region & Date & HMI BR & SOT BR & HMI B & SOT B & $\mu$ \\
\hline 11084 & 20107702 & 0.13 & 0.12 & 2910 & 3130 & 0.93 \\
11164 & 20110301 & 0.16 & 0.16 & 2640 & 2690 & 0.71 \\
11166 & 20110310 & 0.14 & 0.13 & 2680 & 2960 & 0.95 \\
11176 & 20110326 & 0.23 & 0.19 & 2440 & 2690 & 0.78 \\
11339 & 20111106 & 0.09 & 0.09 & 3440 & 3420 & 0.82 \\
\hline
\end{tabular}

a modified center-of-gravity method to determine the magnetic flux density, often called the line-of-sight magnetic field. The advantage of BRs is that intensity data itself can be used as a proxy for maximum umbral field strength across instruments and solar cycles.

Norton et al. (2006) used the Advanced Stokes Polarimeter (ASP) to verify that MDI BR data could be used to infer field strengths. In a similar manner, we use the Hinode Solar Optical Telescope Spectropolarimeter (SOT-SP), which uses a slit and a diffraction grating to sample Stokes I, Q, U and V with a $21.5 \mathrm{~mA}$ spectral resolution in the 6301.5 and $6302.5 \AA$ Fe-I line pair, to verify that HMI BR data can be used to infer field strengths. We chose the active region NOAA 11804 to begin our analysis, as it was observed by Hinode SOT-SP, HMI, and MDI on 2 Jul 2010 near 5:12:00 UT. MDI continuum and line-of-sight magnetograms, and HMI continuum and magnetic field strength products of VSIFV were obtained from JSOC at Stanford University for this date and time, while Hinode SOT-SP continuum and level2 MERLIN (Lites et al., 2007; Ichimoto et al., 2008) magnetic field strength products were obtained from the Community Spectro-polarimetric Analysis Center (CSAC), hosted by HAO NCAR. Figure 5 shows Active Region 11084 continuum intensity, field strength (or flux density in the case of $\mathrm{MDI}$ ), and the scatter plot of intensity versus magnetic field strength as observed by Hinode SOT-SP, HMI and MDI respectively. Due to the difference in resolution between SOT-SP and HMI, data from SOT-SP was binned 2x2 and then interpolated to have the same resolution as HMI of $0.5^{\prime \prime}$. Much of the difference between Hinode SOT-SP

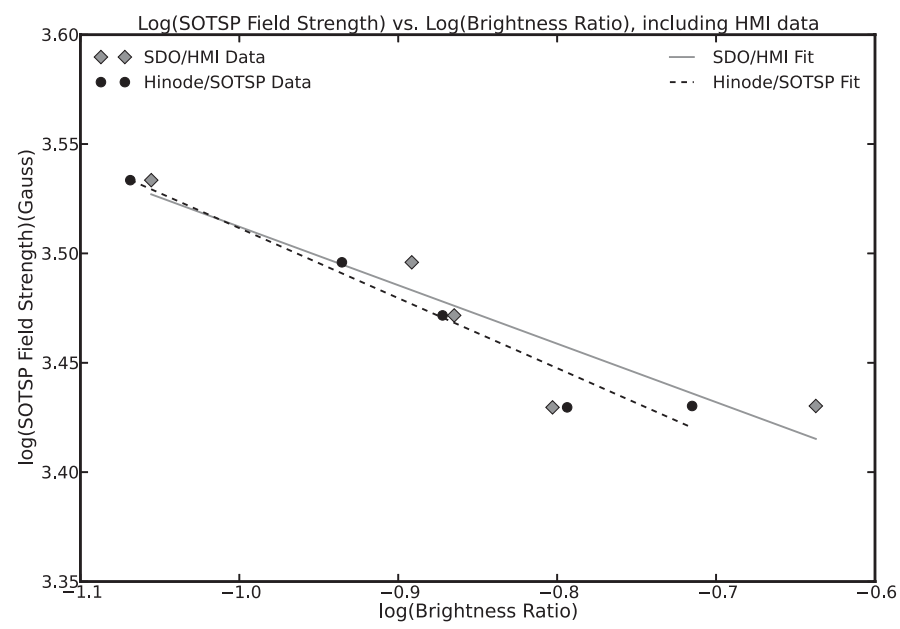

Figure 6. A log plot of SOT-SP field strength versus SOT-SP and HMI brightness ratio, with best-fit lines for each instrument showing very good correlation between the two. 
results and HMI results are due to the fact that the HMI VFISV inversion code assumes the filling fraction of the pixel is one, meaning that all the plasma sampled within the pixel is magnetized and contributing to the Stokes polarization. MDI image quality has degraded significantly over the years. No scattered light corrections are applied.

In order to determine how well Hinode SOT-SP and HMI correlate, we then determined the umbral brightness ratios (BRs) and peak magnetic field strengths of several shared active regions. Thresholding in continuum intensity was used to determine the umbra in each region, and then the darkest pixel in continuum intensity was selected, along with the associated pixel in the accompanying field strength map. The value of the darkest pixel in the continuum intensity was then normalized to the quiet sun to produce a brightness ratio $(\mathrm{Br})$. Table 1 lists each active region, observation date, $\mathrm{BR}$, peak magnetic field strength, and $\mu$ for SOT-SP and HMI. Figure 6 is a log plot of the umbral
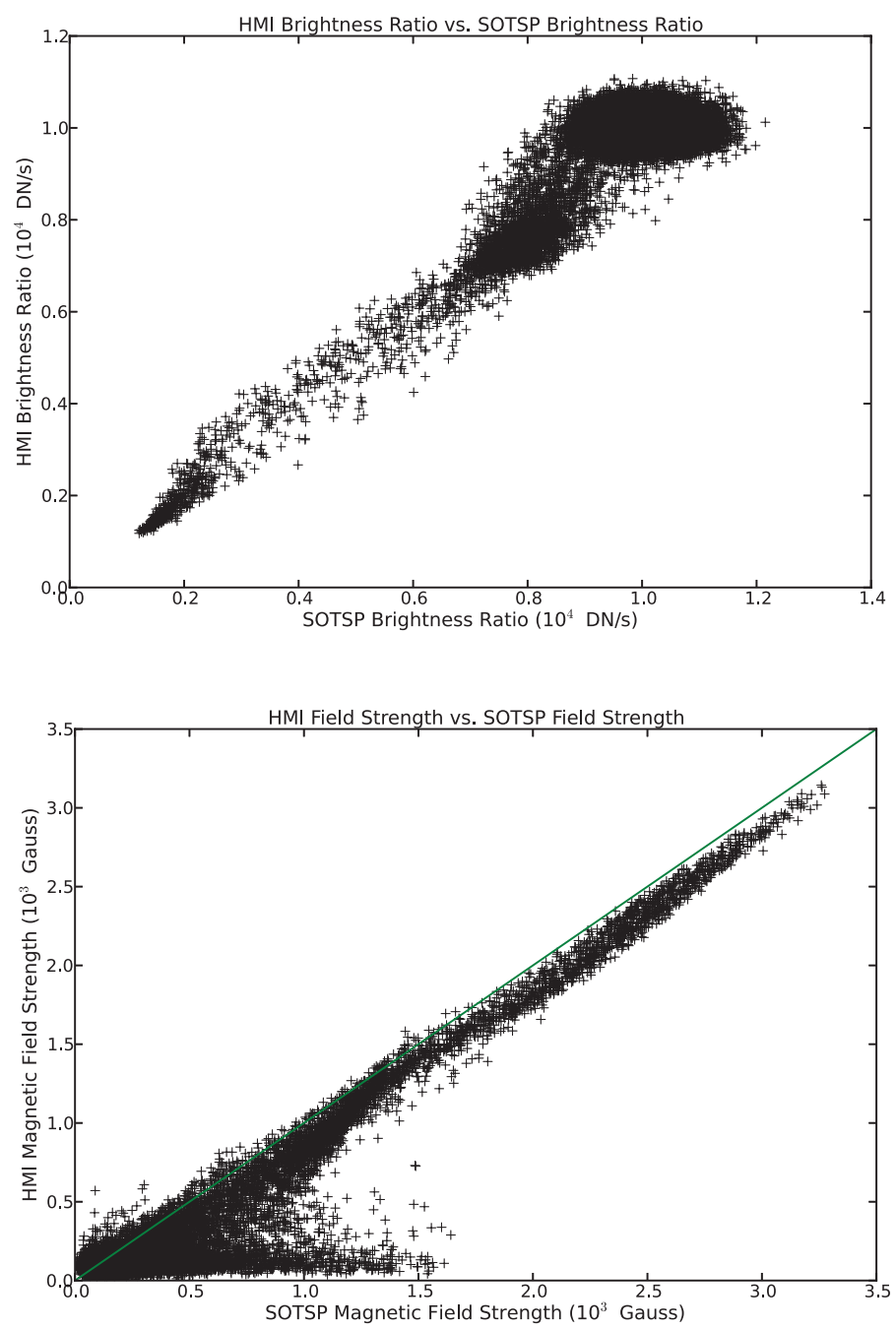

Figure 7. (Top) HMI and SOT-SP brightness ratio data are shown for all points in and surrounding AR 11804 observed on 2 Jul 2010. (Bottom) HMI and SOT-SP magnetic field strength values from the VFISV and MERLIN inversions are compared with a unity line shown. HMI field strengths are slightly lower than SOT-SP in umbra. 
peak magnetic field strengths versus minimum umbral brightness ratios (BRs) for 5 sunspots simultaneously observed in 2010-2012, with linear fits to HMI and SOT-SP data. (Note that the BR has a broad dependence on umbral size.) In this plot, all field strengths are determined by Hinode SOT. We do not use HMI magnetic field data because the HMI algorithm can become saturated in the strongest part of the umbrae depending upon the spacecraft velocity (Liu \& Norton, 2001).

Further comparison between HMI and SOT-SP was carried out by examining scatter plots of the brightness ratio and magnetic field for simultaneous observations of AR11804. As in Figure 5 and 6, SOT-SP data was binned 2x2 and interpolated to the resolution of HMI. The results are plotted in Figure 7. Whereas the scatter is large in the quietSun and weak field regions (when the BR is closer to 1 and the field strength below 1.5 kGauss), the darkest pixels in the umbrae with the strongest magnetic fields compare well. HMI reports slightly weaker field strengths, $\approx 50$ Gauss, for the darkest area of the umbra. Figure 6 and 7 show that the BR of HMI can be used with confidence as a proxy for field strength.

In Figure 8, we show how the intensities vary across the disk for two sunspot regions, AR 11339 and 11429, as observed by HMI and SOT-SP. AR 11339 was observed from 5-11 Nov 2011. AR 11429 was observed from 6-9 Mar 2012 Because limb darkening is observed in the solar atmosphere, it is important to quantify the variations in intensity due to the viewing angle. For our analysis of $\mathrm{BR}$, we use use a continuum intensity Carrington rotation map from HMI that samples sunspots as they cross the central meridian. As most sunspots appear within $30^{\circ}$ of the equator, all sunpot umbral BR data will be determined from $\cos (\theta)=0.87$ or greater. The HMI synoptic continuum intensity data is determined from observations taken every 720 seconds and a fifth order polynomial in $\log (\mu)$ is used to remove limb-darkening. These maps are normalized so that faculae have values above 1.0 and the entire range of the data is from $0.3-1.2$.

In Figure 9, the average BRs and umbral latitudes are plotted for the northern and southern hemispheres. We calculate a mean for all data in each CR and then smooth over $5 \mathrm{CRs}$ for MDI and $3 \mathrm{CRs}$ (due to a shorter time series) for HMI. The top plot shows the well-documented migration of the sunspot latitudes toward the equator. Note that the migration does not proceed that smoothly, but seems to have an asymmetric quality between the two hemispheres. We argue that there is always a need to separate data into north and south hemispheres in order to not blur two dynamo mechanisms that are operating slightly out-of-phase with each other.

\section{Conclusions}

There are some inherent properties of sunspots that are still not understood such as observed rotation rates, the instability of the follower sunspot in a pair, and spacing in longitude. These properties should be studied in more detail, especially as they could be used to distinguish between competing dynamo mechanisms proposed by modelers. Using MDI and HMI brightness ratios (BRs) as a means to determine peak umbral field strengths as calibrated by ASP and SOT-SP, 40 Carrington Rotations into Cycle 23 and 24 , the average peak umbral field strength was 2660 and 2690 Gauss, respectively, as measured by MDI and HMI. The difference is insignificant and as such could be interpreted as Cycle 24 not producing weaker sunspot umbrae even if the sunspot numbers are lower than in Cycle 23. (One caveat, we assume Cycle 24 began in 2009 in CR 2083, whereas others have suggested that the cycle began in 2008, CR 2070. This would change the comparison of the cycle progression.) The Cycle 23 and 24 average latitude of sunspots $40 \mathrm{CR}$ into the cycle was 22 and $17^{\circ}$, respectively, as seen in Figure 9. Sunspot latitude 
data show that the sunspots in both the North and South hemispheres move toward the equator through an antisymmetric motion on an $\approx 9 \mathrm{CR}$ time frame. The progression equatorward of sunspots is not as smooth as might be expected. A consensus is forming that sunspots have darker (stronger) nuclei at sunspot maximum than at the following minimum. However, what is not agreed upon is whether the ascending phase of the cycle produces weaker or stronger sunspots than those at maximum. Therefore, it is not known
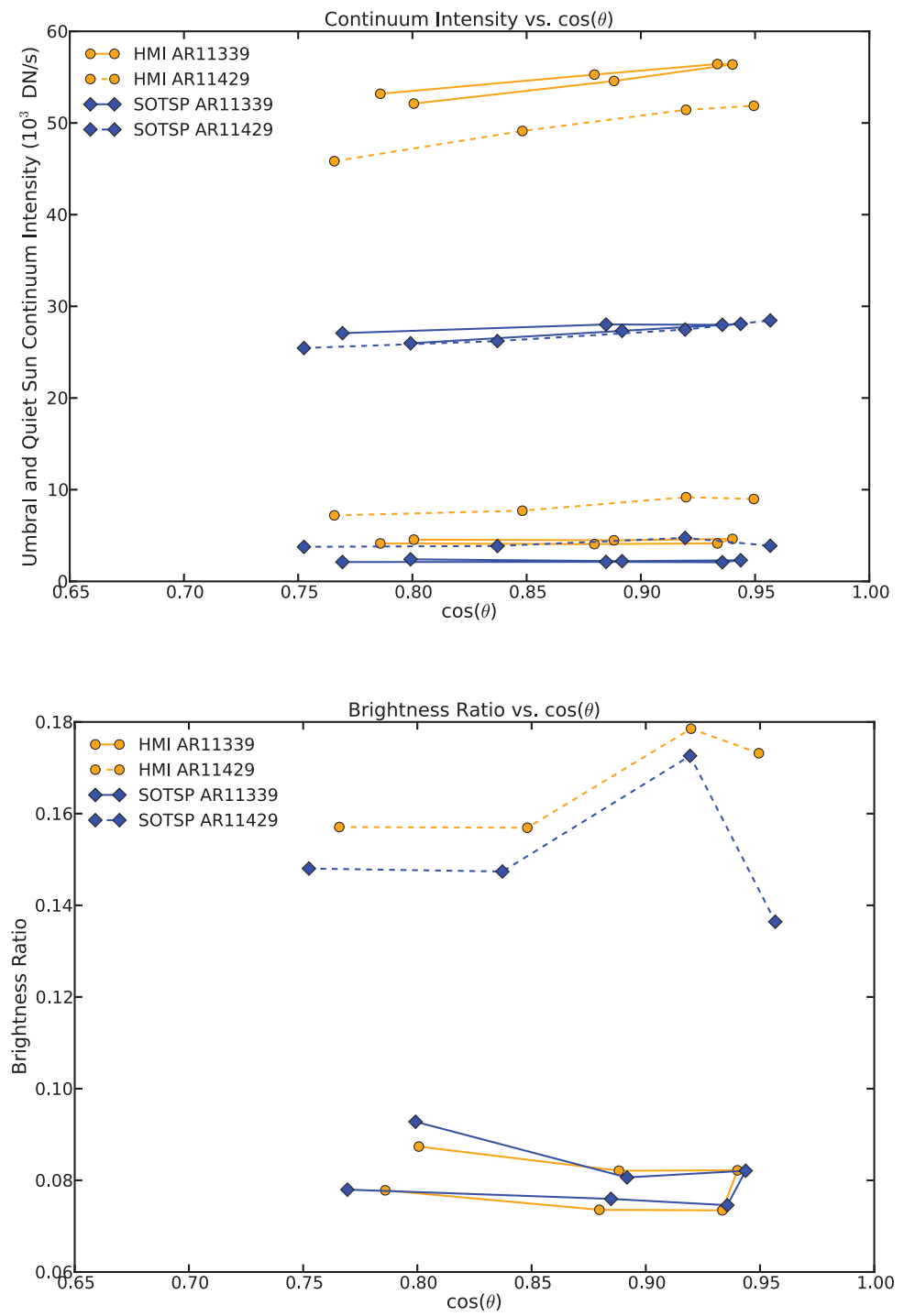

Figure 8. (Top) Observations of continuum intensity versus center-to-limb viewing angle for active regions 11339 and 11429 and their surrounds, as observed by SDO/HMI and Hinode/SOT-SP as these sunspots cross the disk. The higher intensity data at the top and the middle of the plot are the quiet-Sun background intensities while the lower intensities plot are the darkest parts of the umbrae. (Bottom) Observations of bightness ratio versus center-to-limb angle for Active Regions 11339 and 11429 observed by SDO/HMI and Hinode/SOT-SP. The data represents the active regions rotating from the East, through central meridian and toward the West limb. Using Carrington rotation data means that all sunspots are observed at the central meridian and no viewing angle should be lower than 0.85 . 

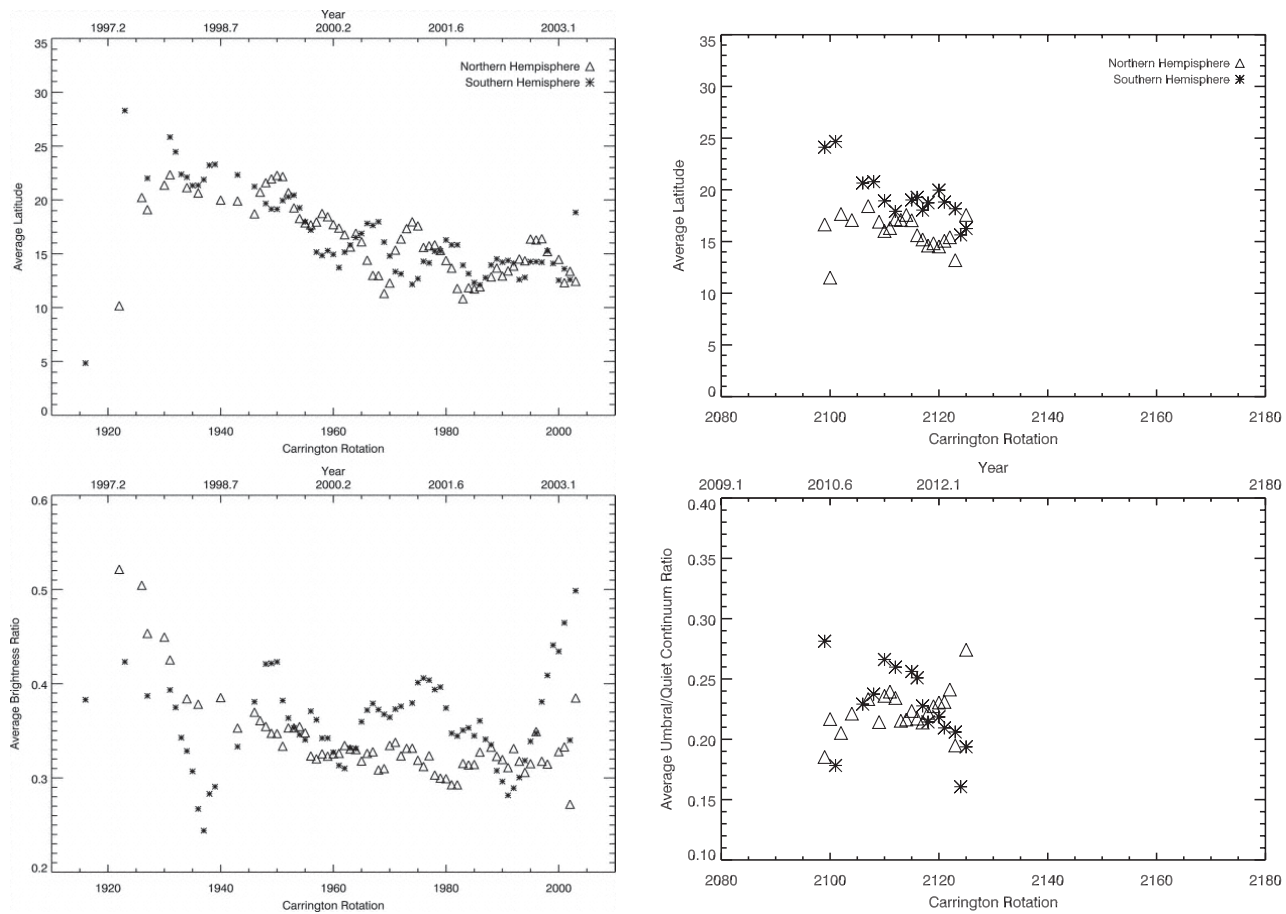

Figure 9. (Left) Cycle 23 sunspot average latitude (top) and minimum average BR data (bottom) as a function of time from 1997 - 2003 as observed by MDI. The data are smoothed for 5 Carrington Rotations and separated into north and south hemisphere. (Right) Cycle 23 sunspot average latitude (top) and minimum average BR (bottom) from 2010-2012 as observed by HMI. The data are smoothed for 3 Carrington Rotations and separated into north and south hemispheres.

whether there is a linear decrease of maximum field strengths over a sunspot cycle or a cyclical increase to maximum and subsequent decrease until minimum.

\section{References}

Babcock, H. W. 1993, ApJ, 133, 572

Beck, J., Duvall, T. L., Jr., \& Scherrer, P. H., 1998, Nature, 394, 653

Beeck, B., Collet, R., Steffen, M., et al., 2012, A\& A, 539, A121

Benevolenskaya, E. E., Hoeksema, J. T., Kosovichev, A. G., \& Scherrer, P. H. 1999, ApJ, 517, L163

Borrero, J. M., Tomczyk, S., \& Norton, A. A., et al., 2007, Sol. Phys., 240, 177

Borrero, J. M., Tomczyk, S., \& Kubo, M., et al. 2011, Sol. Phys., 273, 267

Brandenburg, A. 2005, ApJ, 625, 539

Bray, R. J., \& Loughhead, R. E., 1964, Sunspots, Pitman Press, Bath

Caligari, P., Moreno-Insertis, F., \& Schüssler, M., 1995, ApJ, 441, 886

Caligari, P., Schüssler, M., \& Moreno-Insertis, F., 1998, ApJ, 502, 481

Fan, Y., Fisher, G. H., \& Deluca, E. E., 1993, ApJ, 405, 390

Fan, Y., 2009, Liv. Rev. SolPhys, 6, 4

Ichimoto, K., et al., 2008, Sol Phys., 249, 233

Kosovichev, A. G., et al., 1997, Sol. Phys., 170, 43

Leighton, R. B. 1964, ApJ, 140, 1547

Lites, B. W., et al., 2007, ASP Conference Series, 369, 55

Maltby, P., et al., 1986, ApJ, 306, 284 
Mathew, S. K., et al., 2007, A\&A, 465, 291

Norton, A. A., \& and Gilman, P. A., 2004, ApJ, 603, 348

Norton, A. A., Pietarila Graham, J. D., \& Ulrich, et al., 2006, Sol. Phys., 239, 69

Petrovay, K., 2000, in: Zirin, Ai, Wang (eds), ASP Conference Series, The Magnetic and Velocity Fields of Solar Active Regions, 46, 123

Petrovay, K., 2000, in: A. Wilson (eds), ESA Publications Division and Noordwijk, Netherlands, Proc. of the 1st Solar and Space Weather Euroconference, 463, 3

Penn, M., \& Livingston, W., 2006, ApJ, 649, L45

Rezaei, R., Beck, C., \& Schmidt, W., 2012, A\&A, 541, A60.

Scherrer, P. H., et al., 2012, Sol Phys., 275, 207

Schou, J., Antia, H. M., \& Basu, S., et al. 1998, ApJ, 505, 390

Stein, R. F. \& Nordlund, Å, 2012, ApJ, 753, L13

Watson, F. T., Fletcher, L., \& Marshall, S., 2011, A\&3A, 533, A14

Wilson P. R., Burtonclay D., Li, Y, 1997, ApJ, 489, 395 